\title{
International Council of Museums (ICOM), Répertoire
}

2001, $3^{\mathrm{e}}$ édition, Paris, Comité national français, 156 p.

Yves Laberge

\section{OpenEdition}

\section{Journals}

Édition électronique

URL : http://journals.openedition.org/jso/1216

DOI : $10.4000 /$ jso. 1216

ISSN : $1760-7256$

Éditeur

Société des océanistes

\section{Édition imprimée}

Date de publication : 1 juin 2003

Pagination : 114-115

ISSN : 0300-953x

\section{Référence électronique}


tile transformation des fondements de l'attachement populaire pour le bislama ; à son instrumentalité (vecteur de communication) se voit adjointe une sentimentalité (vecteur de nationalisme) » (p. 258). Il n'y eut aucun choix en faveur du pidgin, il s'imposa faute d'alternative. Seule une poignée de dirigeants tant issus des écoles anglaises que françaises était en mesure de s'exprimer dans une langue européenne. Au sein de chaque parti, pour communiquer avec les militants, encore plus avec les électeurs, ou encore pour convaincre les adversaires politiques, le pidgin s'imposait (faute de mieux). Qu'il soit devenu identitaire, se rapprochant par-là du statut d'un créole, est indéniable mais cela tient à son changement de statut et à la généralisation de son usage : de lingua franca verticale (de Mastas à Boys), il est passé au statut de langue véhiculaire horizontale en usage quasiment entre tous les ni-Vanuatu mais aussi parfois avec les Solomonais et les citoyens de PNG. Tabani remarque que : «La communauté de langue que permet le bislama ne renvoie, toutefois, à aucune communauté de culture (p. 258), -(ce qui le distingue encore d'un créole)-, de plus relève-t-il un paradoxe " cette langue (sans culture propre) contribue (néanmoins) à l'invention d'une kastom nationale » (p. 259).

Contrairement au bislama dont l'essor a plus été accompagné que provoqué, la présence du kava, sa généralisation, sa consommation moderne sont dues à la volonté de Lini et de son premier gouvernement. Tabani écrit p. 261 : « Symbole de pouvoir, le kava l'est resté, mais sous une forme réifiée qui découle de son poids commercial et de son rôle idéologique d'« emblème politique » national. En accord avec ce que souhaitait le grand chef Bongmatur, la constitution a été signée sur une pierre cérémonielle sur laquelle on venait de sacrifier un cochon (Lindstrom, 1997 : 222). Les signataires ont pareillement célébré sa proclamation par des libations de kava, comme c'est désormais le cas lors de toutes les cérémonies officielles » (p. 261). Tabani conclut adroitement ce paragraphe, en l'insérant dans sa problématique générale : «Le kava est devenu un symbole clef de la post-colonialité, et un parfait symbole néo-traditionnel. » (p. 261).

Le dernier avatar de la «kastom polisi » est bien l'ethno-tourisme, il correspond à la volonté de réduire l'« héritage coutumier ancestral» en un «bien culturel ». Tabani dénonce les méfaits locaux de cette dérive affairiste : «En tant que principale industrie et première source de devises, il assure à Vanuatu la pénétration des formes avancées du capitalisme chez des populations ayant conservé jusqu'alors une économie d'auto-subsistance (Tanna, Santo, Pentecôte, Malakula)» (p. 263). Tabani illustre cette commercialisation de la kastom, devenue monopole d'État, à l'aide de diverses activités commercialo-coutumières aux antipodes de la conception première de la coutume, celle d'il y a quelque trente ans.

Cet ouvrage est très bien documenté, tant du point de vue des discours rapportés que des observations de première main ; le plan en quatre parties, unies par un même objet, permet une lecture partielle. La dernière partie, la plus innovante, peut être abordée par toute personne qui, n'ayant pas ou peu de connaissances de l'histoire du pays, veut être informée de la situation contemporaine du Vanuatu.

Certes certaines interprétations peuvent être sujettes à caution (nous les avons signalées), les erreurs flagrantes sont rarissimes : p. 6 : toutes les institutions officielles n'étaient pas dédoublées dans feu le Condominium : poste, marine, aviation, travaux publics ne dépendaient que d'une administration ; p. 257, le pidgin bislama n'a jamais compté aucun lexème d'origine espagnole; les traductions en pidgin et le glossaire auraient mérité d'être discutés. Il s'agit d'un ouvrage remarquable, innovant, très bien écrit, que tout un chacun peut lire avec profit sans être spécialiste de la région.

\section{Jean-Michel CharPenTIER}

LACITO-CNRS

International Council of Museums (ICOM), 2001, Répertoire, 3e édition, Paris, Comité national français, $156 \mathrm{p}$.

Le Comité national français de l'ICOM (acronyme de 1'International Council of Museums) a publié la troisième édition du Répertoire de ses membres. Plus qu'un simple annuaire de muséologues et de chercheurs sur les patrimoines, cet outil de travail permet de mesurer la part importante occupée par le Comité national français au sein de ce gigantesque organisme qu'est l'icom.

Le Répertoire se subdivise en quatre sections et un index : les statuts du Comité national français, les structures de l'ICOM et deux listes, regroupant séparément les membres institutionnels et individuels. Cette section imposante est d'ailleurs conçue comme un annuaire et occupe plus de cent pages, avec les coordonnées de plus de 1600 membres résidant en France. Enfin, un « index par villes » permet de situer selon le lieu et pour chaque institution muséale les membres qui appartiennent à l'ICOM, confirmant que les grandes institutions parisiennes comme le Musée du Louvre, le Museum National d'Histoire Naturelle et la Cité des sciences et de l'industrie comptent un très grand nombre d'adhérents à l'ICOM. En plus des professionnels de la muséologie, on rencontre parmi les membres répertoriés des universitaires, des écrivains, des particuliers, des étudiants. Les notices individuelles se limitent au titre de chaque membre, à ses coordonnées et, dans la moitié des cas seulement, à son adresse électronique.

La partie la plus utile de l'ouvrage regroupe la liste des 28 comités internationaux de l'ICOM, avec l'adresse électronique des responsables respectifs (pp. 18-23). Une brève présentation situe la spécialisation et les objectifs de chaque comité : sur l'audiovisuel, l'éducation, la documentation, les instruments de musique ; d'autres comités se penchent sur la sécurité, les musées littéraires, sur les échanges d'expositions, ou même sur les musées monétaires et bancaires. On comprend que ces comités servent de moteur aux initiatives des membres qui veulent amorcer des collaborations et des échanges. 
Ce Répertoire de professionnels n'est pas destiné au grand public ; il servira principalement d'instrument de référence aux chercheurs et aux bibliothèques spécialisées. Il ne s'agit évidemment pas d'un guide des musées de France ni d'une liste exhaustive de toutes les institutions muséales. La présentation est sobre et l'ouvrage ne comprend pas d'illustrations. On souhaiterait y trouver un index thématique, par exemple des intérêts de recherche des membres. Contrairement à beaucoup de publications de l'ICOM, ce Répertoire indispensable est rédigé exclusivement en français et se consulte aisément.

\section{Yves LABERGE}

Institut québécois des Hautes études internationales Québec, Canada

Hovdhaugen Even, Åshild NAEss and Ingjerg HoËM, 2002. Pileni Texts with a Pileni-English Vocabulary and an English-Pileni Finderlist, Oslo, The Kon-Tiki Museum Occasional Papers 7, 251 p.

Le pileni est un outlier polynésien, parlé par un millier de locuteurs dans différentes petites îles (Pileni, Nifiloli, Matema, Nukapu et Nupani) de la province Temotu, la plus orientale de l'archipel des îles Salomon. Les outliers polynésiens sont des langues polynésiennes parlées en dehors du « triangle polynésien 》délimité par les îles Hawaii, l'île de Pâques et la Nouvelle-Zélande. Ross Clark (1994 : 110) recense 18 outliers, répartis géographiquement en trois groupes : le groupe nord-ouest, à la lisière de la Micronésie (nukuoro, kapingamarangi), le groupe du centre, en Mélanésie, dans les îles Salomon et Santa Cruz (sikaiana, luangiua, pileni, taumako, tikopia et anuta), et le groupe sud-est, en Mélanésie également, auquel appartiennent les outliers du Vanuatu (futuna-aniwa, mele-fila) et celui d'Ouvéa (îles Loyauté), le fagauvea. La grande majorité de ces outliers ont été ou sont encore en contact soit avec des langues d'un autre sous-groupe de la famille océanienne soit avec des langues papoues, comme c'est le cas du pileni, en contact avec l'ayiwo. Il existe encore peu de documents fiables sur ces langues, principalement sur celles parlées dans l'archipel des Salomon. Le présent ouvrage, bien que présenté comme une « version préliminaire » en raison de la situation politique délicate qui rend les enquêtes aux îles Salomon aléatoires, et de l'urgence des besoins d'enseignement en langue vernaculaire, est d'une qualité tout à fait remarquable.

Le livre est divisé en deux parties d'égale importance: une série de textes bilingues pileni/anglais (9-127) et un lexique pileni-anglais assorti d'une brève présentation et d'un index anglais-pileni (129-250).

\section{Les récits lalakhai}

À Pileni, raconter des récits reste encore de nos jours un divertissement favori. Nul n'en a l'exclusivité, et les textes présentés ici ont été enregistrés entre 1997 et 1998 auprès de narrateurs ayant de 12 à 70 ans. Ce sont pour la plupart des récits traditionnels à forte portée symbolique : histoires d'inceste ou de rivalité frèresœur ; quête d'épouses à l'extérieur de son territoire (cf. «La femme de Matema » ou « La fille des étoiles ») provoquant la jalousie des femmes locales; enfant abandonné ou maltraité en quête de justice ou d'autres filiations; actes de vengeance longuement mûris et assortis de pratique magique; mythes de création (apparition de banc de sable ou d'une passe) qui façonnent le paysage en l'humanisant ; explication de nouvelles pratiques (technique de pêche telau à l'aide d'une nasse géante, initiée par une mère qui avait jeté son enfant malformé à la mer).

Certains récits présentent des syncrétismes d'origine occidentale, comme « Le frère et la sœur abandonnés dans la forêt », mélange de Hansel et Gretel et du Petit Poucet (« Ça sent la chair fraîche ici ! »), avec l'évocation d'objets intrus comme l'anneau d'or du fils du roi (« L'anneau perdu ») avalé par une anguille, ou de jumelles spae (de l'anglais spy 'espion'!). L'influence de l'évangélisation se fait aussi sentir dans les deux versions relatant l'histoire du récif (pages 23-30 et 37-38), avec la statufication de deux sœurs, l'une, habillée, tournée vers l'île de Pileni christianisée, l'autre, nue, tournée vers Malaita la païenne.

Les histoires mettant en scène des animaux évoqueront aussi beaucoup de motifs océaniens classiques : bénitier retenant captif sa proie jusqu'à la noyade, à la marée montante; ruse du rat qui réussit à se faire véhiculer en mer ; avantage de la sole tropicale, plate, sur le poisson-coffre quand il s'agit de jouer à cachecache ; solidarité entre la tortue et le héron opposés à la convoitise des hommes.

\section{Lexique}

Le lexique comprend environ 2000 entrées, provenant essentiellement des textes, mais incluant aussi des termes relevés sur le terrain ainsi qu'une liste de poissons conséquente, due au séjour d'un des informateurs pileni à Oslo.

Les entrées appartenant au stock lexical polynésien ancien (la forme reconstruite en proto-polynésien PPN- est parfois donnée à la fin de l'entrée) sont de loin les plus nombreuses, même si leur identification n'est pas toujours évidente, à cause des évolutions phoniques. Ainsi, l'oiseau frégate se dit koraha (PPN *katafa).

Les nombreuses variations de prononciation, en majorité libres, ont posé aux auteurs de gros problèmes de transcription et de choix pour les entrées principales. D'une part, langue de tradition orale, le pileni n'a pas de normes d'écriture. D'autre part, le contact avec les langues des populations installées longtemps avant l'arrivée des Polynésiens a donné lieu, de façon directe ou par accélération de processus d'évolution interne, à des phénomènes d'instabilité accentuelle, provoquant des modifications importantes du système phonologique du pileni: apparition d'une série d'occlusives aspirées (ph, th, kh, lh, mh, nh, gh) parfois écrites par les locuteurs comme des géminées (pp, $\mathrm{tt}, \mathrm{kk}$, etc.) ; apparition de groupes de consonnes (inconnus dans les langues polynésiennes «classi- 